\title{
Artritis tuberculosa del tobillo. Reporte de
} caso

\author{
Tuberculous arthritis of the ankle. Case report \\ Bouchentouf Rachid'
}

VOLUMEN 39 | No 1 | SEPTIEMBRE 2021

FECHA DE RECEPCIÓN: 23/10/2020 FECHA DE APROBACIÓN: 02/06/2021 FECHA PUBLICACIÓN: 16/09/2021

1. Hospital Militar Avicena Jefe del Servicio de Neumología Marrakech - Marruecos

\section{\begin{tabular}{r|l} 
Caso & Clinical \\
Clínico & Case
\end{tabular}}

DOI: https://doi.org/10.18537/RFCM.39.01.06

Correspondencia:

bouchentouf_rachiddyahoo.fr

Dirección:

Hospital Militar Avicena

Código Postal:

40000

Celular:

212661295534

Marrakech - Marruecos

\section{RESUMEN}

Introducción: la tuberculosis del astrágalo no es una patología frecuente, por esta razón se retarda su diagnóstico y tratamiento oportunos, necesarios para evitar la destrucción de las articulaciones y la deformidad esquelética.

Caso clínico: paciente masculino de 45 años de edad presenta traumatismo del tobillo derecho de 5 años de evolución, antecedentes familiares de tuberculosis pulmonar; acude a emergencia por presentar en el tobillo derecho tumefacción dolorosa y fístula cutánea, además de fiebre, sudoración y malestar general. La radiografía y la Tomografía Axial Computarizada (TAC) del pie muestran una lesión osteolítica del astrágalo derecho. Es tratado con antibióticos para estafilococo sin mejorar la sintomatología. Por técnica de punción se toman muestras con resultado negativo en tinción Ziehl-Neelsen, cultivos de aerobios, anaerobios y citología. Se decide el abordaje quirúrgico de la lesión con drenaje. El estudio histopatólogico reveló la presencia de granulomas necrosantes. El paciente evidencia mejora clínica y de seguimiento radiológico 9 meses luego de tratamiento para tuberculosis.

Conclusión: el presente caso indica que en una lesión crónica inflamatoria de tobillo debe descartarse el diagnóstico de tuberculosis sobre todo en pacientes originarios de regiones endémicas.

Palabras clave: artritis infecciosa, tuberculosis osteoarticular, astrágalo, traumatismos de tobillo. 


\section{ABSTRACT}

Introduction: tuberculosis of the talus is not a frequent pathology, for this reason delays in diagnosis and confusion with other processes are highly frequent. Early diagnosis and proper treatment are necessary to avoid joint destruction and skeletal deformity.

Clinical case: It is a case of a 45-years-old male patient presented with a 5-years history of trauma to the right ankle, a family history of pulmonary tuberculosis, who went to the emergency room because of painful swelling and skin fistula in his right ankle, as well as fever, sweating and general malaise. The x-ray and Computed Tomography (CT) of the foot show an osteolytic lesion of the right talus. The patient is treated with antibiotics for staphylococcus without improving symptoms. The wound was punctured, taking samples with a negative result in Ziehl-Neelsen staining, aerobic and anaerobic cultures and cytology. The surgical approach to the lesion with drainage is decided. The histopathological study revealed the presence of necrotizing granulomas. The clinical and radiological evolution was good after 9 months of the treatment for tuberculosis.

Conclusion: this case shows us that in a chronic inflammatory lesion of the ankle the diagnosis of tuberculosis must be ruled out, especially in patients from endemic regions of tuberculosis.

Key words: arthritis infectious, osteoarticular tuberculosis, stragalus, ankle injuries.

\section{INTRODUCCIÓN}

La tuberculosis es un problema de salud pública, siendo una de las principales causas de morbilidad y mortalidad en el mundo, según la Organización Mundial de la Salud (OMS) 10.4 millones de personas desarrollaron tuberculosis en 2016 y 1.7 millones murieron por esta enfermedad ${ }^{1}$. La tuberculosis osteoarticular representa menos del $3 \%$ de los casos de tuberculosis extrapulmonar, las localizaciones más frecuentes son: columna vertebral, cadera o rodilla ${ }^{2,3}$.

La tuberculosis del astrágalo es poco común, solo una decena de casos se han reportado en la literatura ${ }^{4}$. Su diagnóstico es difícil porque tanto los síntomas clínicos como los hallazgos radiológicos son inespecíficos. El retraso en el diagnóstico y el tratamiento tardío pueden ser responsables de secuelas osteoarticulares invalidantes.

\section{PRESENTACIÓN DEL CASO}

Paciente masculino de 45 años de ocupación pastor que vive en una región montañosa cerca de la ciudad de Marrakech, de bajo nivel socioeconómico, con antecedente de traumatismo en tobillo derecho de 5 años de evolución. Su madre fue tratada por tuberculosis pulmonar hace 20 años. 3 meses antes del ingreso presentó dolor del tobillo derecho que aumenta progresivamente de intensidad, con tumefacción y presencia de fístula cutánea (ver Imagen $N^{\circ} 1$ ), acompañado de sensación de alza térmica, sudoración y malestar general.

El paciente recibió tratamiento con antiinflamatorios y antibióticos para estafilococo sin experimentar mejoría.

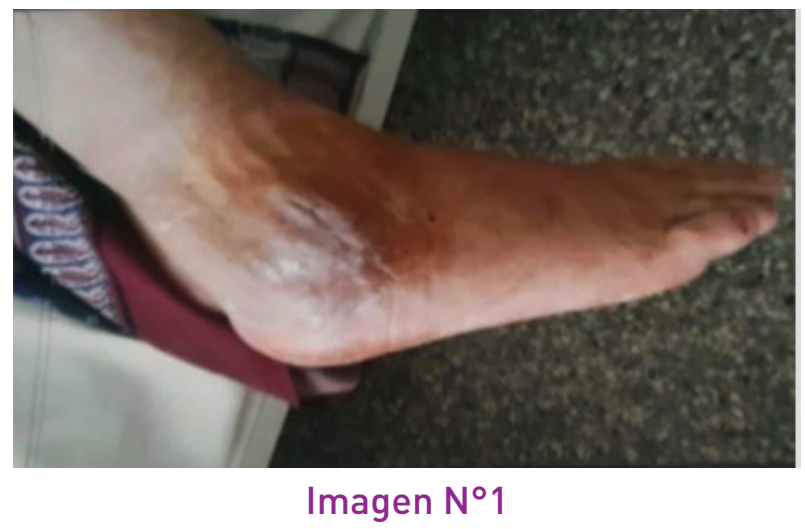

Tumefacción del tobillo derecho 
La exploración física muestra tumefacción dolorosa del tobillo derecho con limitación de su movilidad e incapacidad funcional. La radiografía del tobillo derecho mostró osteopenia con pérdida

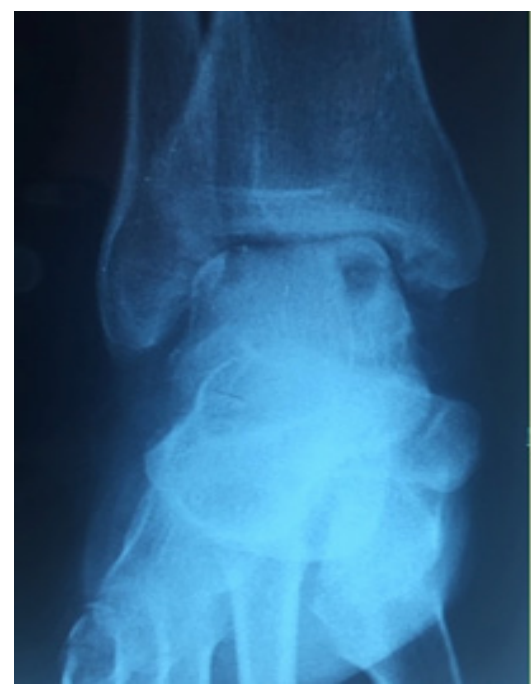

\section{Imagen $\mathrm{N}^{\circ} 2$}

Radiografía simple del tobillo derecho que muestra una lesión osteolítica del astrágalo derecho

En los exámenes paraclínicos se encontró: hemograma con 7500 leucocitos por $\mathrm{mm}^{3}$, neutrófilos $67 \%$, hemoglobina $10.5 \mathrm{~g} / \mathrm{dl}$,plaquetas 235000 por $\mathrm{mm}^{3}$, proteína $C$ reactiva $25 \mathrm{mg} / \mathrm{dl}$. La glicemia, función hepática y renal fueron normales. La prueba cutánea a la tuberculina fue positiva $(15 \mathrm{~mm})$ y la serología para el VIH fue negativa. Se practicó punción-aspiración del espacio articular y erosiones óseas (ver Imagen $\mathrm{N}^{\circ} 2$ ). La TAC del pie mostró proceso osteolítico del astrágalo derecho con ruptura de la cortical y secuestro óseo en su interior (ver Imagen $\mathrm{N}^{\circ} 3$ ).

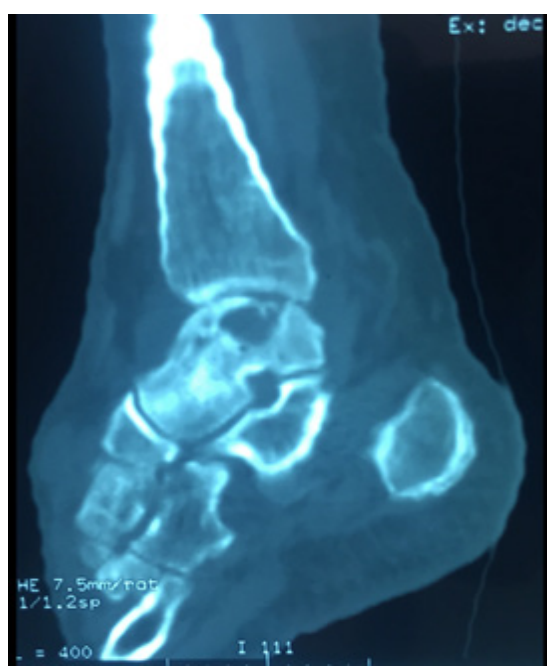

\section{Imagen $\mathrm{N}^{\circ} 3$}

TAC del pie con un proceso osteolítico del astrágalo derecho con ruptura de la cortical y secuestro óseo en su interior

bajo control ecográfico, obteniéndose líquido purulento que al analizar resultó negativo para tinción Ziehl-Neelsen, cultivos de aerobios, anaerobios y citología. Se decidió el abordaje quirúrgico de la lesión con drenaje y toma de muestras, el estudio histopatólogico reveló la presencia de granulomas necrosantes (ver Imagen $\mathrm{N}^{\circ} 4$ ).

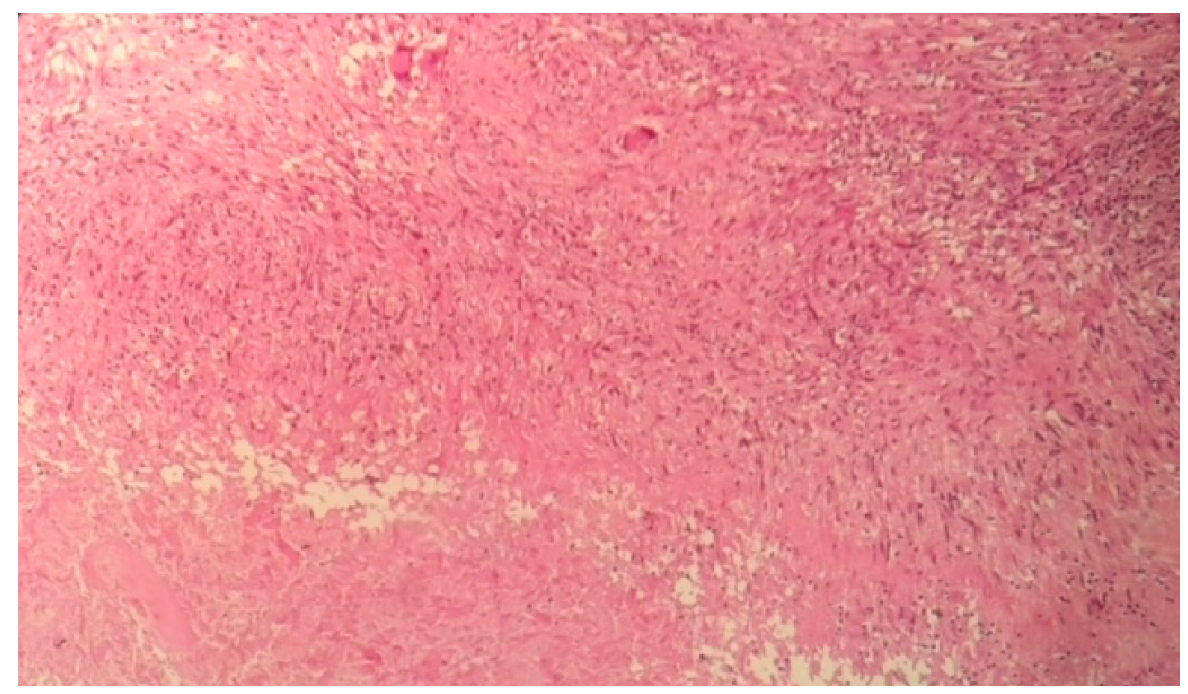

Imagen $\mathrm{N}^{\circ} \mathbf{4}$

Estudio histopatológico con presencia de granulomas necrosantes 
Frente a este resultado se inició tratamiento para tuberculosis con isoniacida $300 \mathrm{mg} / \mathrm{día}$, rifampicina $600 \mathrm{mg} /$ día, pirazinamida $1500 \mathrm{mg} /$ día y etambutol $1200 \mathrm{mg} / \mathrm{día}$, los dos meses seguidos de una terapia combinada de rifampicina e isoniacida hasta completar 9 meses.

Durante los primeros meses se mantuvo soporte sin peso en la extremidad afectada y la articulación del tobillo izquierdo quedo inmovilizada con el yeso. La evolución clínica fue favorable, pero con persistencia de dolor residual y limitación discreta del rango articular astrágalo-tibial.

\section{DISCUSIÓN}

La espondilitis tuberculosa o enfermedad de Pott representa $50 \%$ de los casos de tuberculosis osteomuscular, seguido de la artritis periférica $30 \%$ y grupo menos frecuente $(20 \%)$ constituido por dactilitis, la tenosinovitis y la enfermedad de Poncet ${ }^{5}$.

La tuberculosis del astrágalo, es una localización inusual de tuberculosis incluso en un país endémico de tuberculosis como Marruecos. En la literatura se reportan 12 casos. En la serie Dhillon et al ${ }^{4}$ de 74 pacientes con tuberculosis del pie y del tobillo, la tuberculosis del talud se ha registrado en un solo paciente. Puede ser secundaria a una inoculación directa o como resultado de una diseminación hematógena a partir de un foco esencialmente pulmonar.

La osteoartritis tuberculosa puede ser la única manifestación de la enfermedad o puede presentarse de forma concomitante con afección pulmonar. Los factores que favorecen tal infección pueden ser locales como los traumatismos, o generales, como la inmunodepresión, sobre todo la infección por VIH.

En el diagnóstico diferencial deben considerarse afecciones neoplásicas u osteoartritis por gérmenes piógenos. La presentación clínica es usualmente insidiosa con dolor, inflamación y disminución del rango articular ${ }^{6}$. En fases posteriores se presentan fístulas cutáneas sin aparición de sintomatología sistémica.

Los análisis de laboratorio muestran signos generales de un síndrome inflamatorio, la prueba cutánea a la tuberculina es a menudo positiva. La radiografía estándar del pie es poco específica y puede ser normal al principio, las lesiones encontradas son variables en función de la fase evolutiva de la enfermedad? ${ }^{\text {. }}$.

La TAC muestra la presencia de destrucción ósea, calcificación y secuestro óseo. La Resonancia Magnética Nuclear (RMN) es el examen más sensible que detecta precozmente las anomalías óseas y de los tejidos blandos. El problema de estas formas de tuberculosis radica en la dificultad para llegar a su diagnóstico definitivo, ya que tanto los síntomas clínicos como las pruebas de imagen pueden ser inespecíficos.

Los hallazgos de laboratorio muestran discreta elevación de reactantes como VSG o proteína C reactiva y prueba de Mantoux positiva en la mayoría de los pacientes ${ }^{8}$. Disponiendo actualmente de técnicas de detección de gamma-interferón (IGRA) para en pacientes en los que el Mantoux puede dar falso negativo (como personas inmunosuprimidas y niños $<$ de 5 años) $)^{8}$.

Para la obtención del diagnóstico en la tuberculosis se deben obtener muestras de líquidos y/o tejidos que sean accesibles mediante punción con aguja fina, realizando baciloscopia, cultivo y PCR ${ }^{9}$.

La detección del Bacilo Ácido-Alcohol-Resistente (BAAR) en el estudio directo se obtiene raramente. Los cultivos generalmente en medio sólido de Loweinstein-Jensen no dan colonias visibles hasta las 2 a 4 semanas. El rendimiento de la baciloscopia $y$ cultivos es muy bajo, pues la tuberculosis osteoarticular es una enfermedad paucibacilar.

El estudio histopatológico de tejidos tomados por biopsia muestra los típicos granulomas necrosantes. Su presencia tiene una elevada especificidad y podría justificar la decisión de iniciar tratamiento antituberculoso. En los últimos años se ha detectado ADN de Mycobacterium tuberculosis mediante reacción en cadena de la polimerasa en estas lesiones, aunque de forma inconsistente ${ }^{10}$.

El tratamiento de elección de la tuberculosis del pie es esencialmente clínico, se basa en fármacos antituberculosas durante 6 a 9 meses. Una inmovilización con yeso puede complementar al tratamiento médico. La cirugía puede ser necesaria para obtener un diagnóstico, y como parte del tratamiento con medidas como drenaje de abscesos, limpieza quirúrgica, tratamiento de secuelas ${ }^{11}$. 


\section{CONCLUSIÓN}

La localización de tuberculosis en el pie es poco frecuente. Generando retrasos en el diagnóstico y tratamiento.

En todo proceso de afección osteoatrítica periférica en individuos originarios de una región endémica de tuberculosis se debe considerar el diagnóstico de tuberculosis ósea.

\section{ASPECTOS BIOÉTICOS}

El presente caso clínico fue realizado bajo el consentimiento informado del paciente, con total confidencialidad de datos personales.

\section{INFORMACIÓN DEL AUTOR}

- Bouchentouf Rachid. Doctor en Medicina. Hospital Militar Avicena. Jefe del Servicio de Neumología. Marrakech-Marruecos.

e-mail: bouchentouf_rachid@yahoo.fr

ORCID: https://orcid.org/0000-0003-4839-390X

\section{CONFLICTO DE INTERESES}

El autor declara no tener conflicto de intereses.

\section{FUENTES DE FINANCIAMIENTO}

Autofinanciado.

\section{BIBLIOGRAFÍA}

1. World Health Organization. Global Tuberculosis control WHO report 2016. Suiza: WHO Library; 2016. ISBN 9789241565394.

2. Lasalle-Vignolo A. Tuberculosis ósea en el pie. Caso clínico. Pie Tobillo. 2015; 29(1):3337. DOI: 10.1016/S1697-2198(16)30007-6

3. Tuli SM. General principles of osteoarticular tuberculosis.Clin Orthop Relat Res. 2002, 398:11-9. DOI: 10.1097/00003086200205000-00003

4. Dhillon MS, Aggarwal S, Prabhakar S, et al. Tuberculosis of the foot: an osteolytic variety. Indian J Orthop. 2012, 46(2):206-11. DOI: $10.4103 / 0019-5413.93683$
5. Rubio-Barbón S, Rodríguez-Cocina B, Suárez Del Villar R, et al. Artritis tuberculosa del tobillo.An Med Int (Madrid). 2004;21(9):444446. Disponible en: http://scielo.isciii.es/ scielo.php?script=sci_arttext\&pid=S0212$71992004000900006 \&$ Ing=es.

6. Mjid M, Toujani S, Rekik S, Kanoun L, et al. Ostéo-arthrite chronique de la cheville révélatrice d'une tuberculose pulmonaire. Rev Pneumol Clin. 2016;72/3):214-7. DOI: 10.1016/j.pneumo.2015.11.002

7. Boussel L, Marchand B, Blineau N, et al. Imagerie de la tuberculose ostéo-articulaire. J Radiol. 2002;83(9 pt.1):1025-34. PMID: 12223912

8. Aparicio G, Viudez I, Pérez JL, et al. Tuberculosis osteoarticular de presentación extrapulmonar y extraespinal. Acta Ortopédica Mexicana. 2012;26(1): 15-20. PMID: 23320335

9. Ramírez-Lapausa $M$, Menéndez-Saldaña A, Noguerado-Asensio A. Tuberculosis extrapulmonar, una revisión. Rev Esp Sanid Penit. 2015;17:311. DOI: 10.4321/S157506202015000100002

10. Tan MF, Ng WC, Chan $\mathrm{SH}$, et al. Comparative usefulness of PCR in the detection of Mycobacterium Tuberculosis in different clinical specimens. Journal of Medic Microbiol.1997;46(2): 164-169. DOI: 10.1099/00222615-46-2-164

11. Boussouga $\mathrm{M}$, Tanane $\mathrm{M}$, Bousselmane $\mathrm{N}$, et al. La tuberculose du talus: une localisation rare du bacille de Koch. Rev Chir Orthop Reparatrice Appar Mot. 2002; 88(5):52225. DOI: RCO-09-2002-88-5-0035-1040101019-ART13 